\title{
Fehler bei der Streifenleitungs-Kalibrierung Automatischer Netzwerk-Analysatoren
}

\author{
Errors in Stripline-Calibration of Automatic Network-Analyzers
}

Von Klaus Kindler

Mitteilung aus dem Institut für Hochfrequenztechnik der UNIVERSITÄT HANNOVER

Ubersicht:

Komplexe Streuparameter werden häufig mit dem Automatischen Netzwerk-Analysator der Firma HEWLETT-PACKARD gemessen. Dieses Gerät ist prinzipiell für die Messung koaxialer Komponenten geeignet, und es wird üblicherweise eine koaxiale Kalibrierung durchgeführt. Zur Messung von Streifenleitungs-Komponenten, z.B. Mikrowellentransistoren, bietet allerdings eine Kalibrierung in Streifenleitungstechnik Vorteile. Von den hierfür erforderlichen Standards erweist sich der Absorber als besonders kritisch. In einer Fehler-Analyse wird gezeigt, mit welchen Fehlern bei der Streuparametermessung zu rechnen ist, sofern ein fehlerbehafteter Streifenleitungsabsorber benutzt wird.

Abstract:

The usual measurement system for complex s-parameters is the HEWLETT-PACKARD automatic network analyzer. The internal structure of the network analyzer is coaxial. Therefore this system is suitable for measurement of coaxial components. Coaxial standards are required for calibration. To measure stripline-components like microwave-transistors stripline-calibration seems to be suitable. For calibration stripline-standards are required. Special account has to be taken of the matched termination, which is very critical. In this paper the author presents an investigation of s-parameter-measurement errors involved by an imperfect matched termination.

Für die Dokumentation:

Kalibrierung / Netzwerk-Analysator / Streifenleitungs-MeBtechnik / Streifenleitungssumpf / Streuparametermessung

\section{Einführung}

Das zur Messung komplexer Streuparameter häufig benutzte Meßsystem ist der Automatische Netzwerk-Analysator (ANA) der Firma HEWLETT-PACKARD [1, 2]. Zur Erfassung der immer vorhandenen systematischen Fehler des Netzwerk-Analysators wird üblicherweise das in Bild 1 dargestellte Sechs-Term-Fehlermodell [3] benutzt. Das beim Netzwerk-Analysator HP 8510 benutzte ZwölfTerm-Fehlermodell besteht aus zwei Sechs-Term-Fehlermodellen. Hierbei wird das sonst erforderliche Umdrehen des Meßobjektes vermieden. Die folgenden Untersuchungen haben also auch für Zwölf-Term-Fehlermodelle Gültigkeit.

Das Sechs-Term-Fehlermodell besteht aus zwei FehlerZweitoren. Fehler-Zweitor I wird durch drei Fehlervektoren* beschrieben, Fehler-Zweitor II durch zwei Fehlervektoren. Der Fehlervektor $\underline{e}_{30}$ erfaBt das interne Nebensprechen des Netzwerk-Analysators. Die Bestimmung des Fehlervektors $\underline{e}_{30}$ ist immer unkritisch. Im folgenden wird daher auf die Bestimmung von $\underline{e}_{30}$ nicht weiter eingegangen und diese Größe einfach zu null gesetzt.

Unter der Kalibrierung des ANAs versteht man die Bestimmung der Fehlervektoren. Die Bestimmung der Fehlervektoren des Fehler-Zweitors I wird nach Vorschrift der Firma HEWLETT-PACKARD durch drei Reflexionsmessungen mit den Kalibrierungsstandards „Absorber“, „Referenzkurzschluß“ und „Leerlauf“ bzw. „Offset-Kurzschluß $[1,2]$ durchgeführt. Die Bestimmung der Fehlervektoren des Fehler-Zweitors II erfolgt mit Hilfe einer

\footnotetext{
Der hier und im folgenden verwendete Vektorbegriff ist zwar, mathematisch gesehen, nicht korrekt; er hat sich aber in der Fachliteratur eingebürgert und wurde daher übernommen.
}

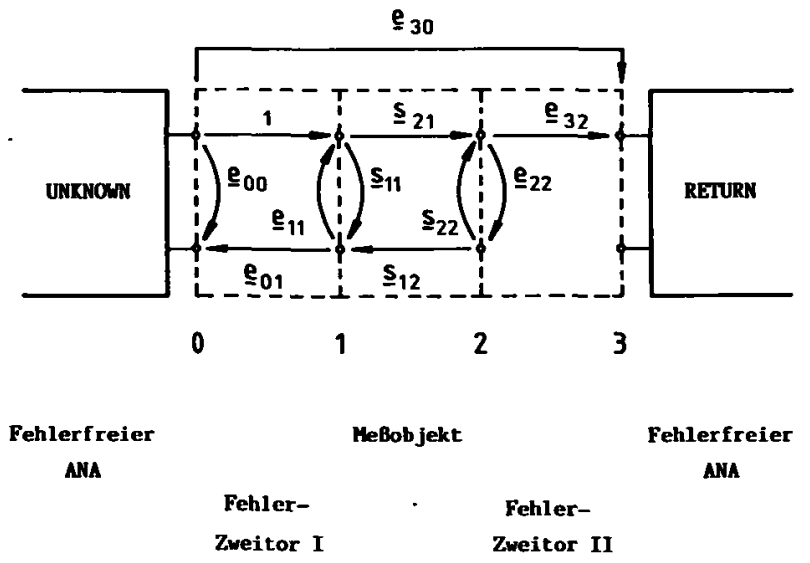

Bild 1: Sechs-Term-Fehlermodell

Die komplexen Größen $\underline{e}_{\mathrm{ik}}$ werden als Fehlervektoren bezeichnet; die komplexen Größen $s_{i k}$ sind die Streuparameter des Meßobjekts.

Durchgangsleitung über eine Reflexions- und eine Transmissionsmessung.

In koaxialer Technik - und auch in Hohlleitertechnik lassen sich derartige Kalibrierungsstandards präzise herstellen, und die Kalibrierung ist unproblematisch. Aus diesem Grund wird der Netzwerk-Analysator in den meisten Fällen für die Messung von Streifenleitungskomponenten, z. B. Transistoren (siehe Bild 2), auch koaxial kalibriert. Hierbei werden die stets vorhandenen Fehler der erforderlichen Koax-Streifenleitungs-Übergänge vernachlässigt, wodurch erhebliche Meßfehler entstehen können, wie bereits vom Verfasser in [4] gezeigt. 


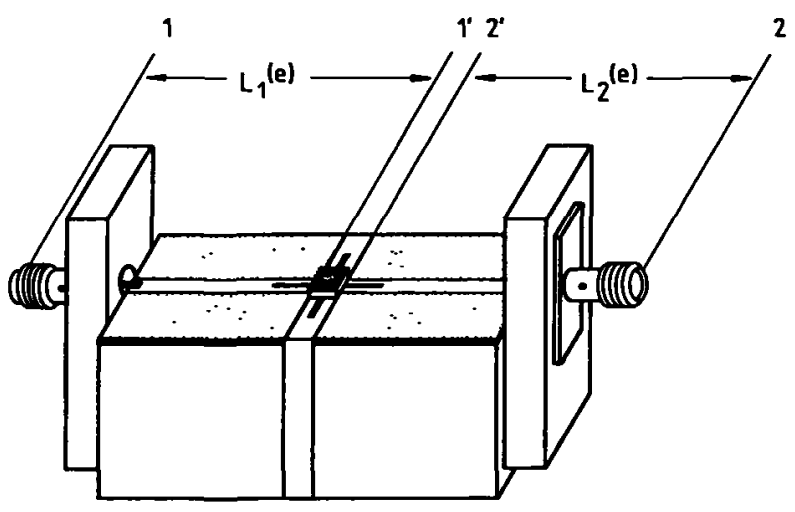

Bild 2: Typische Anordnung zur Streuparameter-Messung von Mikrowellentransistoren mit Streifenleitungsanschlüssen Die angegebenen Längen $L^{(e)}$ stellen elektrische Längen dar.

Eine Erfassung der Fehler der Koax-StreifenleitungsÜbergänge zusammen mit den Fehlern des ANAs ist nur durch eine vollständige Streifenleitungs-Kalibrierung möglich. Hierbei bestehen jedoch erhebliche Probleme, da präzise Streifenleitungs-Kalibrierungsstandards nur äuBerst schwierig hergestellt werden können. Bei einer vollständigen Streifenleitungs-Kalibrierung ist also immer mit einer fehlerhaften Kalibrierung zu rechnen, die natürlich Auswirkungen auf die Meßgenauigkeit hat.

Die folgende Untersuchung wird nun zeigen, ob die koaxiale Kalibrierung und anschließende Benutzung fehlerbehafteter Übergänge oder die vollständige Streifenleitungs-Kalibrierung mit fehlerbehafteten Standards zu geringeren Meßfehlern führt.

\section{Streifenleitungsstandards}

Die zur vollständigen Streifenleitungs-Kalibrierung erforderlichen Standards sind prinzipiell in Bild 3 dargestellt. Der Kurzschluß sowie der Pseudo-Leerlauf - häufig wird hierfür ein Offset-Kurzschluß verwendet, in seltenen Fällen auch eine leerlaufende Streifenleitung (z. B. [5]) - lassen sich noch gut realisieren. Der Absorber dagegen ist äußerst schwierig herzustellen. Mit guten Chip-Widerständen aus $\mathrm{NiCr}$ können bis $15 \mathrm{GHz}$ breitbandig $20 \mathrm{~dB}$ Rückflußdämpfung erreicht werden, mit speziellen Streifenleitungsausführungen [5] sogar $30 \mathrm{~dB}$, allerdings nur schmalbandig. In [6] ist ein breitbandiger Absorber beschrieben worden, der bis $15 \mathrm{GHz}$ sogar $25 \mathrm{~dB}$ Rückflußdämpfung aufweist und bis $18 \mathrm{GHz}$ noch immer oberhalb $20 \mathrm{~dB}$ liegt. Auch Streifenleitungs-Schiebelasten sind realisiert worden (z. B. [7, 8]). Hierzu werden Blöcke aus stark verlustbehafteten Materialien sehr hoher Dielektrizitätskonstante benutzt, die auf der Streifenleitung bewegt werden. Eine offensichtlich hochwertige, mechanisch jedoch aufwendige Streifenleitungs-Schiebelast ist in [9] beschrieben. Leider fehlt dort eine Angabe über die erzielte effektive Richtschärfe, mit der die Güte der Schiebelast beurteilt werden könnte.

$\mathrm{Da}$ in erster Linie offensichtlich der Streifenleitungsabsorber fehlerbehaftet ist, werden für die folgenden Betrachtungen alle anderen Kalibrierungsstandards, wie Kurzschluß, Leerlauf bzw. Offset-Kurzschluß und Durchgangsleitung, als fehlerfrei betrachtet.

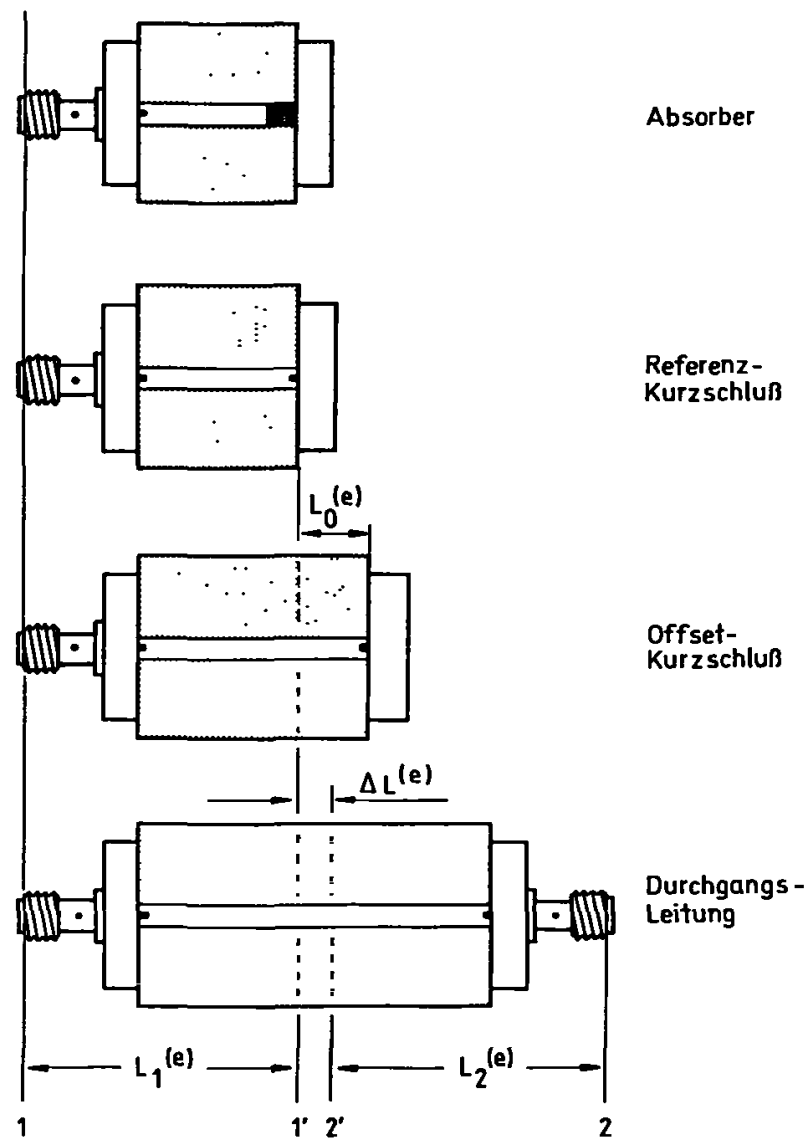

Bild 3: Streifenleitungs-Kalibricrungsstandards (Absorber, Referenz-Kurzschluß, Offset-Kurzschluß und Durchgangsleitung)

3. Kalibrierungsfehler durch einen fehlerbehafteten Absorber

Ziel der vollständigen Streifenleitungs-Kalibrierung ist eine resultierende Beschreibung der Fehler-Zweitore des ANAs zusammen mit den Übergängen. Daher werden sinnvollerweise die Referenzebenen direkt in die Meßobjektebenen, z. B. in die Transistorebenen $1^{\prime}$ und $2^{\prime}$, gelegt (Bild 2 und 3).

Wird für die Kalibrierung ein nichtidealer Absorber mit dem endlichen Reflexionsfaktor $\Delta \underline{I}_{\mathrm{A}}$ oder eine Schiebelast benutzt, die nach einer Kreisformungsprozedur die $\mathbf{A b}$ weichung $\Delta \underline{I}_{\mathrm{A}}$ vom tatsächlichen Kreismittelpunkt liefert, so ergibt die Reflexionsmessung (z.B. nach [10]) den fehlerbehafteten Fehlervektor $\underline{e}_{00}^{(\mathbf{F})}$ :

$$
\underline{r}_{1}=\underline{e}_{00}^{(\mathrm{F})}=\underline{e}_{00}+\frac{\underline{e}_{01} \Delta \underline{\Gamma}_{\mathrm{A}}}{1-\underline{e}_{11} \Delta \underline{\Gamma}_{\mathrm{A}}}
$$

Die Reflexionsmessung mit einem fehlerfreien Kurzschluß, der auch die Referenzebene 1' festlegt, ist durch

$$
\underline{r}_{2}=\underline{e}_{00}-\frac{\underline{e}_{01}}{1+e_{11}}
$$

beschrieben, und die Reflexionsmessung mit einem als ideal angenommenen Leerlauf des Reflexionsfaktors $1 / \underline{0}^{\circ}$ führt auf

$$
\underline{r}_{3}=\underline{e}_{00}+\frac{\underline{e}_{01}}{1-\underline{e}_{11}}
$$


Aus den durchgeführten Reflexionsmessungen berechnet der ANA $[1,2]$ die Fehlervektoren $\underline{e}_{11}^{(\mathrm{F})}$ und $\underline{e}_{01}^{(\mathrm{F})}$, die aufgrund des Fehlers von $\underline{e}_{00}^{(\text {F) }}$ natürlich auch fehlerbehaftet sind. Nach einer kurzen Rechnung ergibt sich

$$
\underline{e}_{11}^{(\mathrm{F})}=\frac{\underline{r}_{3}+\underline{r}_{2}-2 \underline{e}_{00}^{(\mathrm{F})}}{\underline{r}_{3}-\underline{r}_{2}}
$$

Dies geht mit (1), (2) und (3) schließlich über in den fehlerbehafteten Fehlervektor

$$
\underline{e}_{11}^{(\mathrm{F})}=\frac{\underline{e}_{11}-\Delta \underline{\Gamma}_{\mathrm{A}}}{1-\underline{e}_{11} \Delta \underline{I}_{\mathrm{A}}}
$$

Für den dritten Fehlervektor des Fehler-Zweitors I ergibt sich

$$
\underline{e}_{01}^{(\mathrm{F}}=\left(\underline{e}_{00}^{(\mathrm{F})}-\underline{r}_{2}\right)\left(1+\underline{e}_{11}^{(\mathrm{F})}\right)
$$

und daraus mit (1), (2) und (5) schließlich

$$
\underline{e}_{01}^{(F)}=\frac{\underline{e}_{01}\left(1-\Delta \underline{\Gamma}_{A}^{2}\right)}{\left(1-\underline{e}_{11} \Delta \underline{\Gamma}_{A}\right)^{2}} \text {. }
$$

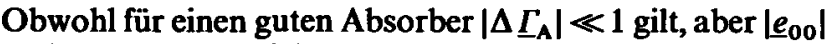
und auch $\left|e_{11}\right|$ üblicherweise in der gleichen Größenordnung wie $\left|\Delta \underline{\Gamma}_{\mathrm{A}}\right|$ liegen, können $\underline{e}_{00}^{(\mathrm{F})}$ und $\underline{e}_{11}^{(\mathrm{F})}$ offensichtlich mit erheblichen Fehlern behaftet sein, wie (1) bzw. (5) deutlich zeigen. Dagegen unterscheidet sich der Fehlervektor $\underline{e}_{01}^{(\mathrm{F})}$ nach (7) wegen $\left|\Delta \underline{\Gamma}_{\mathrm{A}}^{2}\right| \ll 1$ und $\left|\underline{e}_{11} \Delta \underline{\Gamma}_{\mathrm{A}}\right| \ll 1$ nur unwesentlich vom richtigen Wert $\underline{e}_{01}$, dessen Betrag üblicherweise in der Größenordnung eins liegt.

Um die Fehlervektoren des Fehler-Zweitors II zu bestimmen, wird eine Durchgangsleitung eingefügt (Bild 3). Der ANA mißt damit (z. B. nach [10]) den Reflexionsfaktor

$$
\underline{r}_{4}=\underline{e}_{00}+\frac{\underline{e}_{01} \underline{e}_{22} \mathrm{e}^{-\mathrm{j}\left\{2 \beta \Delta L^{(0)\}}\right.}}{1-\underline{e}_{11} \underline{e}_{22} \mathrm{e}^{-j\left(2 \beta \Delta L^{(0)}\right)}},
$$

wobei festgesetzt wird, daß der Fehlervektor $\underline{e}_{22}$ in der Ebene 2' liegen soll (Bilder 2 und 3). Außerdem mißt der ANA den Transmissionsfaktor

$$
\underline{t}_{5}=\frac{\underline{e}_{32} \mathrm{e}^{-\mathrm{j}\left(\beta \Delta L^{(0)}\right)}}{1-\underline{e}_{11} \underline{e}_{22} \mathrm{e}^{-\mathrm{j}\left(2 \beta \Delta L^{(0)}\right)}} .
$$

Hierbei stellt $\boldsymbol{\beta}$ die Phasenkonstante dar.

Aus den Meßwerten berechnet der ANA die Fehlervektoren des Fehler-Zweitors II, die ebenfalls fehlerbehaftet sind. Nach einer kurzen Rechnung erhält man

$$
\underline{e}_{22}^{(\mathrm{F})}=\frac{\left(r_{4}-\underline{e}_{00}^{(\mathrm{F})}\right) \mathrm{e}^{\mathrm{j}\left(2 \beta \Delta L^{(0)}\right)}}{\underline{r}_{4} \underline{e}_{11}^{(\mathrm{F})}+\underline{e}_{01}^{(\mathrm{F})}-\underline{e}_{00}^{(\mathrm{F})} \underline{e}_{11}^{(\mathrm{F})}}
$$

und mit (1), (5), (7) und (8) schlieBlich

$$
\underline{e}_{22}^{(\mathrm{F})}=\frac{\underline{e}_{22}-\Delta \underline{\Gamma}_{\mathrm{A}} \mathrm{e}^{+\mathrm{j}\left\{2 \beta \Delta L^{(0)}\right\}}}{1-\underline{e}_{22} \Delta \underline{\Gamma}_{\mathrm{A}} \mathrm{e}^{-\mathrm{j}\left\{2 \beta \Delta L^{(e)}\right\}}} .
$$

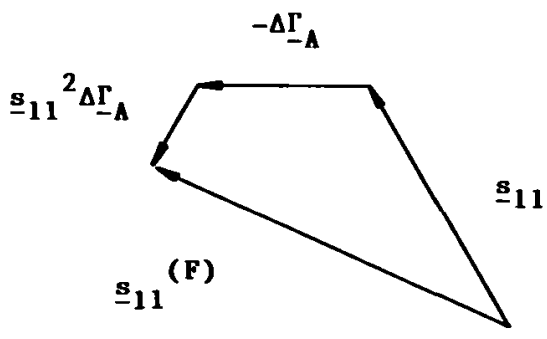

Bild 4: Fehlerhaft bestimmter Streuparameter $\underline{\underline{1}}_{11}^{(\mathrm{f})}$ als Folge eines Kalibrierungsabsorber-Fehlers
Für $\underline{e}_{32}^{(\mathrm{F})}$ erhält man

$$
\underline{e}_{32}^{(\mathrm{F})}=\frac{\underline{t}_{5}\left(1-\underline{e}_{11}^{(\mathrm{F})} \underline{e}_{22}^{(\mathrm{F})} \mathrm{e}^{-\mathrm{j}\left\{2 \beta \Delta L^{(0)}\right)}\right)}{\mathrm{e}^{-\mathrm{j}\left[\boldsymbol{\beta} \Delta L^{(0)}\right\}}},
$$

woraus sich mit (5), (9) und (11)

$$
\underline{e}_{32}^{(F)}=\frac{\underline{e}_{32}\left(1-\Delta \underline{\Gamma}_{A}^{2}\right)}{\left(1-\underline{e}_{11} \Delta \underline{\Gamma}_{\mathrm{A}}\right)\left(1-\underline{e}_{22} \Delta \underline{\Gamma}_{\mathrm{A}} \mathrm{e}^{-\mathrm{j}\left(2 \beta \Delta L^{(0) 3}\right)}\right.}
$$

ergibt.

Wie $\underline{e}_{01}^{(\mathrm{F})}$, so ist auch $\underline{e}_{32}^{(\mathrm{F})}$, dessen Betrag üblicherweise in der Größenordnung von eins liegt, wegen $\left|\Delta \Gamma_{\Lambda}^{2}\right| \ll 1$, $\left|e_{11} \Delta \underline{\Gamma}_{\mathrm{A}}\right| \ll 1$ und $\left|e_{22} \Delta \underline{\Gamma}_{\mathrm{A}}\right| \ll 1$ mit nur sehr geringen Fehlern behaftet, während $\underline{e}_{22}^{\mathrm{f}}$, dessen Betrag in der GröBenordnung von $\left|\Delta \underline{\Gamma}_{\mathrm{A}}\right|$ liegt, analog zu $\underline{e}_{00}^{(\mathrm{F})}$ und $\underline{e}_{11}^{(\mathrm{F})}$ erhebliche Fehler aufweisen kann.

\section{MeBfehlerabschätzung}

\subsection{Reflexionsmessungen bei rückuirkungsfreiem Vierpol}

Liegt als Meßobjekt ein Zweipol oder auch ein rückwirkungsfreier Vierpol mit dem Reflexionsfaktor $\underline{s}_{11}$ vor, so mißt der ANA den Reflexionsfaktor

$$
\underline{r}_{1 \mathrm{~m}}=\underline{e}_{00}+\frac{\underline{e}_{01} \underline{s}_{11}}{1-\underline{e}_{11} \underline{s}_{11}} \text {. }
$$

Mit diesem Meßwert und mit den fehlerbehafteten Fehlervektoren berechnet der ANA den fehlerbehafteten Reflexionsfaktor $\underline{s}_{11}^{(\mathrm{F})}$

$$
\underline{s}_{11}^{(\mathrm{F})}=\frac{\underline{r}_{1 \mathrm{~m}}-\underline{e}_{00}^{(\mathrm{F})}}{\underline{e}_{01}^{(\mathrm{F})}+\underline{e}_{11}^{(\mathrm{F})}\left(\underline{r}_{1 \mathrm{~m}}-e_{00}^{(\mathrm{F})}\right)} .
$$

Daraus ergibt sich nach Einsetzen von (1), (5), (7) und (14) schließlich

$$
\underline{s}_{11}^{(\mathcal{F})}=\frac{\underline{s}_{11}-\Delta \underline{\Gamma}_{\mathrm{A}}}{1-\underline{s}_{11} \Delta \underline{\Gamma}_{\mathrm{A}}}
$$

Gleichung (16) wird dadurch bestätigt, daß die Reflexionsfaktoren $\underline{s}_{11}=-1$ und $\underline{s}_{11}=1$ - dies sind die Reflexionsfaktoren der verwendeten Kalibrierungsstandards Kurzschluß und Leerlauf - unabhängig von der Fehlergröße $\Delta \underline{\Gamma}_{\mathrm{A}}$ richtig wiedergegeben werden.

Es sei bemerkt, daß (16) auch über die bilineare Transformation [11] abgeleitet werden kann. Der hier gewählte Weg läßt jedoch die Entstehung von Meßfehlern an Hand des Berechnungsmodus innerhalb des ANAs erkennen.

Eine Reihenentwicklung von (16) (geometrische Reihe), die nach dem linearen Glied wegen $\left|\underline{s}_{11} \Delta \underline{\Gamma}_{\mathrm{A}}\right| \ll 1$ bereits abgebrochen werden kann,

$$
\underline{s}_{11}^{(\mathrm{F})}=\left(\underline{s}_{11}-\Delta \underline{\Gamma}_{\mathrm{A}}\right)\left(1+\underline{s}_{11} \Delta \underline{\Gamma}_{\mathrm{A}}+\cdots\right),
$$

führt nach Ausmultiplikation und Vernachlässigung des Summanden $\underline{s}_{11} \Delta \underline{\Gamma}_{\mathrm{A}}^{2}\left(\left|\underline{s}_{11} \Delta \underline{\Gamma}_{\mathrm{A}}^{2}\right| \ll 1\right)$ auf

$$
\underline{s}_{11}^{(\mathrm{P})}=\underline{s}_{11}-\Delta \underline{\Gamma}_{\mathrm{A}}+\underline{s}_{11}^{2} \Delta \underline{\Gamma}_{\mathrm{A}}+\cdots \text {. }
$$

Gleichung (18) ist in der komplexen Ebene in Bild 4 dargestellt. Für Reflexionsfaktorwinkel von

$$
\operatorname{Arc}\left\{\underline{s}_{11}\right\}= \pm 90^{\circ}
$$

haben die fehlererzeugenden Vektoren die gleiche Richtung, so da $B$ in diesen Fällen mit den maximalen Winkeloder Betragsfehlern zu rechnen ist. Dagegen sind die fehlererzeugenden Vektoren für die Fälle Arc $\left\{s_{11}\right\}=0^{\circ}$ und Arc $\left\{\underline{s}_{11}\right\}= \pm 180^{\circ}$ entgegengesetzt gerichtet, so da $B$ dann die kleinsten Fehler vorliegen. 
Für die Reflexionsfaktorbeträge von $\left|\Sigma_{11}\right|=1$ ist der Fehler dann sogar null, wie bereits anhand der exakten Gleichung (16) festgestellt worden ist.

Wird für die Rückflußdämpfung des Kalibrierungsabsorbers $\Delta \underline{\Gamma}_{\mathrm{A}}$ die Übergangs-Rückflußdämpfung $\underline{\ddot{u}}_{11}$ gesetzt, weist (18) die gleiche Form wie (6) in [4] auf, und Bild 4 entspricht Bild 3 in [4], sieht man von den Phasenbeziehungen einmal ab. Da für die Berechnung der maximal möglichen Phasen- und Betragsfehler ohnehin nur die Beträge der fehlererzeugenden Vektoren von Bedeutung sind, ist die in [4] (Abschnitt 3.1) vom Verfasser bereits durchgeführte Fehlerabschätzung auch für die vollständige Streifenleitungs-Kalibrierung gültig und bedarf keiner weiteren Erläuterung. Der Kurvenparameter der Bilder 4a, b in [4], die die maximal möglichen ReflexionsMeBfehler bei rückwirkungsfreiem Vierpol darstellen, ist jedoch nicht als Übergangs-Rückflußdämpfung $\left|\underline{\ddot{u}}_{11}\right|_{(\mathrm{dB})}$, sondern als $A$ bsorber-Rückflußdämpfung $\left|\Delta \underline{\Gamma}_{\mathrm{A}}\right|_{(\mathrm{dB})} \mathrm{zu}$ interpretieren.

\subsection{Allgemeine Vierpolmessungen}

Die Meßfehlerabschätzung läßt sich hierfür sehr leicht anschaulich durchführen. Hierzu muß sowohl die Ebene 1' als auch die Ebene 2' (Bild 2 bzw. 3) betrachtet werden.

In der Ebene 2 ' sind die fehlerhaft bestimmten Fehlervektoren $\underline{e}_{22}^{(\mathrm{F})}$ und $\underline{e}_{32}^{(\mathrm{F})}$ von Bedeutung. Da von diesen jedoch nur $\underline{e}_{22}^{(\mathrm{F})}$ (siehe (11)) wesentlich vom richtigen Wert abweichen kann, bedarf lediglich dieser Vektor einer genaueren Betrachtung. Eine Reihenentwicklung von (11) analog zu (16) und (17) führt auf

$$
\underline{e}_{22}^{(P)}=\underline{e}_{22}-\Delta \underline{\Gamma}_{\mathrm{A}} \mathrm{e}^{\mathrm{j}\left(2 \beta \Delta \Delta L^{(0)}\right)}+\cdots,
$$

wobei wegen $\left|\underline{e}_{22} \Delta \underline{I}_{\mathrm{A}}\right| \ll 1$ keine weiteren Summanden berücksichtigt werden müssen. Da $\underline{\underline{e}}_{22} \mid$ in der Größenordnung von $\left|\Delta \underline{\Gamma_{A}}\right|$ liegt, ist $\underline{e}_{22}$ praktisch unbestimmbar, wie (19) zeigt. Gleichung (19) läßt sich dahingehend interpretieren, daB $\underline{e}_{22}$ infolge eines fiktiven Ubergangs mit dem Reflexionsfaktorbetrag $\left|\Delta \underline{\Gamma}_{\hat{A}}\right|$ in der Ebene 2 ' falsch gemessen worden ist.

Bezüglich der Ebene 1' kann (18) wegen des äquivalenten Aufbaus zu (6) in [4] so interpretiert werden, daB auch hier ein fiktiver Übergang des Reflexionsfaktorbetrages $\left|\Delta \underline{\Gamma}_{\mathrm{A}}\right|$ vorhanden ist.

Es ist offensichtlich, daß das nun vorliegende Problem äquivalent dem der Abschnitte 3.2 und 4 in [4] ist, so daB die dort durchgeführten Fehlerabschätzungen für Reflexions- und Transmissionsmessungen auch für die vollständige Streifenleistungs-Kalibrierung uneingeschränkt Gültigkeit haben und keiner weiteren Erläuterung bedürfen. Der Kurvenparameter der Bilder 6a, b in [4] (ReflexionsMeßfehler) und der Bilder 8a, b in [4] (TransmissionsMeßfehler) ist allerdings als Rückflußdämpfung des verwendeten Kalibrierungsabsorbers $\left|\Delta \underline{\Gamma}_{\mathrm{A}}\right|_{(\mathrm{dB})} \mathrm{zu}$ interpretieren.

\section{Vergleich zweier Kalibrierverfahren und} Zusammenfassung

Die durchgeführte Fehlerbetrachtung erlaubt nun einen Gütevergleich zwischen der vollständigen StreifenleitungKalibrierung und der in [4] untersuchten Methode, bei der der ANA koaxial kalibriert wird unter anschließender Benutzung fehlerbehafteter Koax-Streifenleitungs-Übergänge. Der Gütevergleich wird dadurch vereinfacht, da $\mathrm{B}$ die Fehlerbeschreibung beider Kalibrierungs-Modifikationen äquivalent ist.

Die vollständige Streifenleitungs-Kalibrierung würde nur dann zu geringeren Meßfehlern führen, wenn sich Absorber-Rückflußdämpfungen erreichen ließen, die erheblich über den Rückflußdämpfungen liegen würden, die sich mit Koax-Streifenleitungs-Übergängen realisieren lassen. Dies ist aber nicht der Fall. $\mathrm{Zu}$ verwirklichen sind Übergangs-Rückflußdämpfungen zwischen $20 \mathrm{~dB}$ und $30 \mathrm{~dB}$. Diese Werte liegen in den gleichen Größenordnungen, die auch den erreichbaren Absorber-Rückflußdämpfungen entsprechen.

Unter Berücksichtigung der Realisierbarkeitsgrenzen läßt sich global feststellen, daß die vollständige Streifenleitungs-Kalibrierung aufgrund des unpräzisen Kalibrierungsabsorbers nicht zu geringeren Meßfehlern führt als die koaxiale Kalibrierung unter Benutzung fehlerhafter Koax-Streifenleitungs-Übergänge.

Der Verfasser dankt Herrn Prof. Dr.-Ing. J. Marquardt für die Unterstützung dieser Arbeit.

\section{Literetur:}

[1] Hackborn, R. A.: An automatic network analyzer system. Microwave Journal 11 (1968) 2, S. 16-19.

[2] Hand, B. P.: Developing accuracy specifications for automatic network analyzer systems H.JP-Joural 21 (1970) 2, S. $16-19$.

[3] Fitzpatrick, J.: Error modek for systems measurement. Mierowave Journal 21

[4] Kindler, K.: Abschštzung der Fehler bei der Streuparameter-Messung von Streifenleitungs-Komponenten mit Hilfe Automatischer Netzwerk-Amalysatoren. Frequenz

[5] Khill (1987) H. 6/7, S. 168-172.

Impedances improves device charecterisation high-power GaAs FET terminating S. 255-263.

[6] Fischer, W.; Wiesbeck, W.: Wideband microstrip termination. Nachr.techn. Z. 26 (1973) $2,583-85$

[7] Sieprath, W.; Llbricht, J.: Mefverfahren zur Bestimmung von MIC-Parametern (1974) 5 , S. 161- 163 . [8] Riech, V.: Melprogramm und Eichstandards fir Microstrip-Messungen mit einem [9] Ootomatischen Netzmerk-Analysator. Nachr.techn, Z 24 (1971) 5, S. 255-259.
S. 295-301. Hartmann, T.: Microstrip slidiag load. Frequenz 36 (1982) i1,

[10] Brandt, H.: Schaltungsklehre linearer Mikrowellennetze. Stuttgart: Hirzel-Verlag. 1970. [11] Kasa, I.: Closed-form mathematical solutions to some network analyzer calibration
equations. IEEE IM-23 (1974) 4, S. 399-402.

Dr.-Ing. Kbaus Kindler, kabelmetal electro GmbH - Antennenproduktion -, Kabelksmp 20, D-3000 Hannover 1

(Eimgegangen am 7. 1. 1987) 